\title{
THE STRUCTURE OF ALTERNATIVE DIVISION RINGS
}

\author{
R. H. BRUCK AND ERWIN KLEINFELD
}

1. Introduction. A ring $R$ is said to be alternative if $(x x) y=x(x y)$, $(y x) x=y(x x)$ for all $x, y$ of $R$. And $R$ is a division ring if it has a nonzero element and the equations $a x=b, y a=b$ have unique solutions $x, y$ for $a \neq 0$; the existence of a unit is not postulated.

Let $R$ be an alternative ring without divisors of zero. ${ }^{1}$ If $a, b$ are nonzero elements of $R$ and $n$ is a rational integer, the equation ( $n a) b$ $=a(n b)$ shows that $n a=0$ if and only if $n b=0$. Therefore we can assign a characteristic (zero or prime) to $R$ in the usual manner. The centre $C$ of $R$ is the subring consisting of all $c$ in $R$ such that $c x=x c$, $c(x y)=(c x) y=x(c y)$ for all $x, y$ of $R$. If $C$ contains a nonzero element, define the central quotient ring $R / / C$ as the set of all formal fractions $x / c, x \in R, c \in C, c \neq 0$, with the obvious definitions: (i) $x / c=y / d$ if and only if $d x=c y$; (ii) $(x / c)+(y / d)=(d x+c y) /(c d)$; (iii) $(x / c)(y / d)$ $=(x y) /(c d)$. It is readily verified that $R / / C$ is an alternative ring with unit $c / c$ and with centre $F=C / / C$, the quotient field of $C$. Especially in view of (i), the mapping $x \rightarrow(c x) / c$ is an isomorphism of $R$ into $R / / C$, and we imbed $R$ in $R / / C$ by making the identifications $x$ $=(c x) / c$. These remarks allow us to state the main theorem of the paper:

TheOREM A. Let $R$ be a not-associative, alternative ring without divisors of zero and of characteristic not 2 . Then the centre $C$ of $R$ contains a nonzero element and $R$ may be imbedded in $R / / C$, which is an alternative division algebra of order 8 ( $a$ so-called Cayley-Dickson algebra) over its centre $F=C / / C$.

Corollary 1. If, in addition, $C$ is a field, $R$ is a Cayley-Dickson algebra over $C$.

COROLLARY 2. Every alternative division ring of characteristic not 2 is either (i) a field or skew-field or (ii) a Cayley-Dickson algebra over its centre.

COROLlaRY 3. Every ordered alternative ring is associative.

Theorem A seems of less immediate interest than Corollaries 2, 3. As we shall point out explicitly in $\$ 5$, the latter have important applications to projective planes. However, at no point does the proof of

\footnotetext{
Presented to the Society, November 25, 1950; received by the editors December 9 , 1950.

1 We adopt the convention that 0 is not a divisor of zero.
} 
Theorem A (as compared with that of Corollary 2) raise additional difficulties of any significance, and indeed we are spared several times the necessity of dealing with division subrings rather than subrings. The methods are new in the main and Lemma 2.2 appears to be the only result which is entirely known. It has been our policy to adopt in each lemma the weakest hypotheses which will allow its proof without introducing complications unnecessary for the proof of Theorem A. The paper is relatively self-contained; except in $\$ 5$ and Appendix I, the only essential reference is to a paper by A. A. Albert [2]. ${ }^{2}$

It is perhaps worth noting that a direct proof of Corollary 3 may be based upon $\$ 2$, Lemmas $3.1,4.1$, the definition of $R / / C$, and the remark that an ordered ring cannot contain a quaternion algebra.

2. Elementary properties. In any ring $R$ (not necessarily alternative) the commutator $(x, y)$ and the associator $(x, y, z)$ are defined by

$$
(x, y)=x y-y x, \quad(x, y, z)=x y \cdot z-x \cdot y z,
$$

for all $x, y, z$ of $R$. By direct substitution,

$$
\begin{aligned}
& (x y, z)-x(y, z)-(x, z) y=(x, y, z)-(x, z, y)+(z, x, y) \\
& (w x, y, z)-(w, x y, z)+(w, x, y z)=w(x, y, z)+(w, x, y) z
\end{aligned}
$$

Henceforth let $R$ be alternative. The defining relations for $R$ may be written as $(x, x, y)=0,(y, x, x)=0$. Using the linearity of the associator, we find $0=(x+y, x+y, z)=(x, x, z)+(x, y, z)+(y, x, z)$ $+(y, y, z)=(x, y, z)+(y, x, z)$ and, similarly, $0=(x, y+z, y+z)$ $=(x, y, z)+(x, z, y)$. Therefore

$$
(x, y, z)=-(y, x, z)=-(x, z, y) .
$$

From (2.4) and the fact that $(x, x, y)=0$ follows

$$
(x, x, y)=(x, y, x)=(y, x, x)=0 .
$$

We shall say that a (multi-)linear function $h\left(x_{1}, x_{2}, \cdots, x_{n}\right)$ from $R$ to $R$ is skew-symmetric provided it takes the value 0 whenever at least two of the $x$ 's are equal. Thus (2.5) expresses the skew-symmetry of the associator. Just as (2.5) implies (2.4), so any skew-symmetric function changes sign when two of its arguments are interchanged. Note that the commutator is skew-symmetric. In view of (2.4), (2.2) can be rewritten as

$$
(x y, z)=x(y, z)+(x, z) y+3(x, y, z) .
$$

\footnotetext{
2 Numbers in brackets refer to the bibliography at the end of the paper.
} 
Next we introduce the (linear) function $f(w, x, y, z)$ by

$$
(w x, y, z)=(x, y, z) w+x(w, y, z)+f(w, x, y, z) .
$$

LEMMA 2.1. In an alternative ring $R$, the function $f$ defined by (2.7) is skew-symmetric and satisfies the identities

$$
\begin{aligned}
3 f(w, x, y, z)= & (w,(x, y, z))-(x,(y, z, w)) \\
& +(y,(z, w, x))-(z,(w, x, y)), \\
f(w, x, y, z)= & ((w, x), y, z)+((y, z), w, x) .
\end{aligned}
$$

Proof. By (2.4), (2.3) can be written as (wx, y,z)-(xy,z,w) $+(y z, w, x)=w(x, y, z)+(w, x, y) z$. Substitution from (2.7) gives (2.10) $f(w, x, y, z)-f(x, y, z, w)+f(y, z, w, x)=F(w, x, y, z)$,

where $F(w, x, y, z)$ denotes the right-hand side of (2.8), and hence changes sign when $w, x, y, z$ are permuted cyclically. Thus, from (2.10), $0=F(w, x, y, z)+F(x, y, z, w)=f(w, x, y, z)+f(z, w, x, y)$, or

$$
f(w, x, y, z)=-f(z, w, x, y) .
$$

Hence $f$ changes sign when its arguments are permuted cyclically (by (2.11)) and when the last two are interchanged (by (2.7)), and therefore when any two are interchanged. Since $f(w, x, y, y)=0, f$ is skewsymmetric. In particular, (2.10) reduces to (2.8). Subtract from (2.7) the result of interchanging $w$ and $x$, and get $((w, x), y, z)$ $=-(w,(x, y, z))+(x,(y, z, w))+2 f(w, x, y, z)$. Computed thus, the right-hand side of (2.9) reduces to the left-hand side in view of (2.8). This completes the proof.

From Lemma 2.1 we deduce a number of well known but useful identities:

Lemma 2.2. For all $x, y, z$ of an alternative ring $R$, we have

$$
\begin{aligned}
& \left(x^{2}, y, z\right)=x(x, y, z)+(x, y, z) x, \\
& (x, x y, z)=(x, y, x z)=(x, y, z) x, \\
& (x, y x, z)=(x, y, z x)=x(y, y, z),
\end{aligned}
$$

and the Moufang identities

$$
\begin{gathered}
x y \cdot z x=x(y z \cdot x), \\
x(y \cdot x z)=(x y \cdot x) z, \quad(z x \cdot y) x=z(x \cdot y x) .
\end{gathered}
$$

Proof. For (2.12), set $w=x$ in (2.7). Again, by (2.7), (xy, z, x) $=(y, z, x) x+y(x, z, x)+f(x, y, z, x)=(x, y, z) x$, giving (2.13); similarly for (2.14). As for (2.15), $x y \cdot z x=x(y \cdot z x)+(x, y, z x)=x(y \cdot z x)$ 
$+x(y, z, x)=x(y z \cdot x)$, by (2.14). The first equation of (2.16) comes by $(x y \cdot x) z=x y \cdot x z+(x y, x, z)=x(y \cdot x z)+(x, y, x z)-(x, x y, z)$ $=x(y \cdot x z)$; and the second, similarly.

If $h\left(x_{1}, \cdots, x_{n}\right)$ is any function from $R$ to $R$ and if $A_{i}$ $(i=1, \cdots, n)$ are subsets of $R$, we denote by $h\left(A_{1}, \cdots, A_{n}\right)$ the set of all elements $h\left(a_{1}, \cdots, a_{n}\right)$ with $a_{i}$ in $A_{i}$. A subset consisting of a single element will usually be denoted by that element. At various points in the paper we shall use without comment the known fact that any two elements $a, b$ of an alternative ring $R$ are contained in an associative subring of $R$. Proofs (in the notation just explained) of this and other "associativity" theorems will be found in Appendix I.

The following lemma helps to motivate the proof of an essential result:

Lемма. 2.3. In an alternative ring $R$, the (linear) function $g(u, v, w, x, y)$, defined for all $u, v, w, x, y$ of $R$ by

$$
\begin{aligned}
f(u v, w, x, y)= & u f(v, w, x, y)+f(u, w, x, y) v+(u, x, y)(v, w) \\
& +(u, w)(v, x, y)+g(u, v, w, x, y),
\end{aligned}
$$

is skew-symmetric in $u, v, w$ and in $x, y$. Equivalently,

$$
\begin{aligned}
& f(u v, u, x, y)=u f(v, u, x, y)+(u, x, y)(v, u), \\
& f(u v, v, x, y)=f(u, v, x, y) v+(u, v)(v, x, y),
\end{aligned}
$$

for all $u, v, x, y$ of $R$.

Proof. Since $f$ is linear, so is $g$. And $g$ is zero for $y=x$, hence skewsymmetric in $x, y$. Write $G(u, v, w)=g(u, v, w, x, y)$ for fixed $x, y$. Then (2.18) states that $G(u, v, u)=0$, or that $G$ is skew-symmetric in $u, w$. Similarly (2.19) states that $G$ is skew-symmetric in $v, w$. Assuming these, we have $G(u, u, w)=-G(u, w, u)=0$, so that $G$ is skew-symmetric in $u, v, w$. To prove (2.18), note that $f(v u, u, x, y)$ $=((v u, u), x, y)+((x, y), v u, u)$, by (2.9). However, $((v u, u), x, y)$ $=((v, u) u, x, y)=u((v, u), x, y)+(u, x, y)(v, u)+f((v, u), u, x, y)$, by (2.6), (2.7); and $((x, y), v u, u)=u((x, y), v, u)$, by (2.14). Adding and using (2.9), we get $f(v u, u, x, y)=u f(v, u, x, y)+(u, x, y)(v, u)$ $+f(v u-u v, u, x, y)$; this is equivalent to (2.18). To prove (2.19) we operate similarly on $f(v u, v, x, y)$. One can also obtain expressions for $g$ which exhibit the correct symmetries.

The next two lemmas deal with new and essential identities.

Lemma 2.4. For all $x, y, z$ of an alternative ring $R$,

$$
((x, y, z), x, y)=(x, y)(x, y, z)=-(x, y, z)(x, y),
$$




$$
\left((x, y, z)^{2}, x, y\right)=0 .
$$

Proof. Writing $u=(x, y, z), v=(x, y)$, we apply Lemma 2.2 in two ways to $p=\left(x^{2}, y, z y\right)$. On the one hand, $p=x(x, y, z y)+(x, y, z y) x$ $=x \cdot y u+y u \cdot x$. On the other hand, $p=y\left(x^{2}, y, z\right)=y \cdot x u+y \cdot u x$. By subtraction, $0=x \cdot y u-y \cdot x u+(y, u, x)=x y \cdot u-(x, y, u)-y x \cdot u$ $+(y, x, u)+(y, u, x)=(x, y) u-(u, x, y)$. Therefore $(u, x, y)=v u$. Similarly, $\left(x^{2}, y, y z\right)=x \cdot u y+u y \cdot x=x u \cdot y+u x \cdot y$ and hence $(u, x, y)$ $=-u v$. This proves (2.20). Thence $\left(u^{2}, x, y\right)=u(u, x, y)+(u, x, y) u$ $=u \cdot v u+(-u v) u=0$, proving (2.21). Note that also $\left(u^{2}, x, z\right)$ $=\left(u^{2}, y, z\right)=0$.

LEMMA 2.5. Let $x, y, z$ be any elements of an alternative ring $R$. Then: (i) $\left((x, y, z)^{4}, x\right)=0$; (ii) if $(x, y, z)$ is not a divisor of zero, $\left((x, y, z)^{2}, x\right)$ $=0$.

Proof. Write $u=(x, y, z), v=u^{2}, w=f(x, y, z, v)$. From (2.18), $f(x v, x, y, z)=x f(v, x, y, z)+(x, y, z)(v, x)=-x w+u(v, x)$. With a similar use of (2.19), we have

$$
u(v, x)=x w+f(x v, x, y, z), \quad(v, x) u=w x+f(v x, x, y, z) .
$$

Using (2.17) and omitting some zero terms, we find $f(x v, y, x, z)$ $=x f(v, y, x, z)+(x, y)(v, x, z)+g(x, v, y, x, z)$. However, $(v, x, z)=0$ by (2.21), and hence $f(x v, x, y, z)+x w=g(v, x, y, x, z)$. Similarly, $f(v x, x, y, z)+w x=-g(v, x, y, x, z)$. Comparison with (2.22) yields

$$
u(v, x)=-(v, x) u .
$$

By (2.7), $f(x, y, x v, z)=(x y, x v, z)-(y, x v, z) x-y(x, x v, z)$. Also $(x, x v, z)=(x, v, z) x=0$ by $(2.13),(2.21)$, so that

$$
f(x v, x, y, z)=(x v, y, z) x-(x v, x y, z) .
$$

By (2.7), (2.21), $(x v, y, z)=(v, y, z) x+v(x, y, z)+f(x, v, y, z)=v u+w$. By (2.7), (2.13), $(x v, x y, z)=(v, x y, z) x+v(x, x y, z)+f(x, v, x y, z)$ $=(x y, z, v) x+v \cdot u x-f(x y, x, z, v)$. Since $(v, u, x)=\left(u^{2}, u, x\right)=0$, (2.24) yields

$$
f(x v, x, y, z)=[w-(x y, z, v)] x+f(x y, x, z, v) .
$$

The term in brackets is zero; indeed $(x y, z, v)=y(x, z, v)+(y, z, v) x$ $+f(x, y, z, v)=w$, by (2.7), (2.21). Also, by (2.18), (2.21), $f(x y, x, z, v)$ $=x f(y, x, z, v)+(x, z, v)(y, x)=-x w$. Therefore (2.25) gives $f(x v, x, y, z)=-x w$. Hence, by (2.22), (2.23),

$$
u(v, x)=0 \mathrm{~J}=(v, x) u .
$$


If $u=0$, items (i), (ii) are trivial, so assume $u \neq 0$. If $u$ is not a divisor of zero, (2.26) yields (ii). In any case, since $v=u^{2},\left(u^{4}, x\right)=\left(v^{2}, x\right)$ $=v(v, x)+(v, x) v=0$ by $(2.6),(2.26)$. This completes the proof.

3. The centre. The nucleus $N$ of an alternative ring $R$ is the set of all $n$ in $R$ such that $(n, R, R)=0$. In this terminology, the centre $C$ of $R$ is the set of all $c$ in $R$ such that $(c, R)=(c, R, R)=0$. In particular, $C \subset N$. A subset $S$ of $R$ is said to generate $R$ if no proper subring of $R$ contains $S$.

Lemma 3.1. Let $R$ be an alternative ring with centre $C$, and let $S$ be a generating subset of $R$. Then the element $p$ of $R$ is in $C$ if and only if $(p, S)=0=(p, S, S)$. And, incidentally, $C$ is a subring of $R$.

Proof. In one direction the proof is obvious. Therefore let $p$ satisfy $(p, S)=(p, S, S)=0$. Let $X$ be the set of all $x$ in $R$ such that $(p, S, x)=0$. Clearly $X$ is closed under subtraction. Also, by (2.7), (2.9), $\left(x^{\prime} x, p, s\right)=x\left(x^{\prime}, p, s\right)+(x, p, s) x^{\prime}+f\left(x^{\prime}, x, p, s\right)=f\left(x^{\prime}, x, p, s\right)$ $=\left(\left(x^{\prime}, x\right), p, s\right)+\left((p, s), x^{\prime}, x\right)=\left(\left(x^{\prime}, x\right), p, s\right)=\left(x^{\prime} x, p, s\right)-\left(x x^{\prime}, p, s\right)$. Hence $(X X, p, S)=0, X$ is a subring of $R$. Since $(p, S, S)=0, X$ contains $S, X=R,(p, S, R)=0$. Next let $Y$ be the set of all $y$ in $R$ such that $(p, y, R)=0$. Then $0=\left(y x, y^{\prime}, p\right)=x\left(y, y^{\prime}, p\right)+\left(x, y^{\prime}, p\right) y$ $+f\left(y, x, y^{\prime}, p\right)=-f\left(y, y^{\prime}, x, p\right)$ and hence $\left(y y^{\prime}, x, p\right)=y^{\prime}(y, x, p)$ $+\left(y^{\prime}, x, p\right) y+f\left(y, y^{\prime}, x, p\right)=0$. As before, we deduce that $Y=R$, $(p, R, R)=0$. Finally, let $Z$ be the set of all $z$ in $R$ such that $(p, z)$ $=0$. By (2.6), $\left(z z^{\prime}, p\right)=z\left(z^{\prime}, p\right)+(z, p) z^{\prime}+3\left(z, z^{\prime}, p\right)=0$. Thus, again, $Z=R,(p, R)=0$. Hence $p$ is in $C$. In the same manner, we verify that $C$ is a subring.

Lemma 3.2. Let $R$ be an alternative ring with nucleus $N$. Let $a, b$ be elements of $R$ such that $(a, b, R)=0$. Then: (i) $(a, b) \in N$; (ii) if $(a, b)$ is neither zero nor a divisor of zero, $a, b \in N$; (iii) if $n \in N$ is neither zero nor a divisor of zero, $n x \in N$ implies $x \in N$.

Proof. By (2.7), (2.9), for $x, y$ in $R, 0=(x y, a, b)=y(x, a, b)$ $+(y, a, b) x+f(x, y, a, b)=((x, y), a, b)+((a, b), x, y)=((a, b), x, y)$. Hence $((a, b), R, R)=0$, proving (i). In particular, $(N, R) \subset N$. For $n \in N, x, y, z \in R,(n x, y, z)=x(n, y, z)+(x, y, z) n+f(n, x, y, z)$ $=(x, y, z) n+((n, x), y, z)+((y, z), n, x)=(x, y, z) n$. Hence $(n x, R, R)$ $=(x, R, R) n$, and (iii) is clear. By Lemma 2.2, $(a, b, R)=0$ implies $(a, b a, R)=0$. Therefore, by (i), $N$ contains $(a, b a)=(a, b) a$. If $(a, b)$ $\neq 0$ is not a divisor of zero, we see from (i) and (iii) that $a$ is in $N$. Similarly for $b$. This completes the proof.

THEOREM 3.1. Let $R$ be an alternative ring without divisors of zero, with nucleus $N$, centre $C$. Then either $N=R$ or $N=C$. 
Corollary. If also $(R, R, R) \neq 0$, then $(a, b, R)=0$ implies $(a, b)=0$.

Proof. Assume $N \neq R$. Then there exists at least one $x$ of $R$ which is not in $N$. If $n \neq 0$ is in $N, n x$ is not in $N$, by Lemma 3.2 (iii). For any $n^{\prime} \in N,\left(n^{\prime}, x\right)$ and $\left(n^{\prime}, n x\right)$ are not divisors of zero. Therefore, by Lemma 3.2 (ii), $\left(n^{\prime}, x\right)=0=\left(n^{\prime}, n x\right)$. Hence, by (2.6), $0=\left(n x, n^{\prime}\right)$ $=n\left(x, n^{\prime}\right)+\left(n, n^{\prime}\right) x+3\left(n, x, n^{\prime}\right)=\left(n, n^{\prime}\right) x$. Now $x \neq 0$, since 0 is in $N$. Hence $\left(n, n^{\prime}\right)=0,(n, N)=0$. Thus $(n, R)=0$ for $n \neq 0$. Since $(0, R)$ $=0,(N, R)=0$. Hence $N \subset C \subset N$, or $N=C$. As for the corollary, if $(R, R, R) \neq 0$, then $N \neq R$, so $N=C$. Suppose that $(a, b) \neq 0$. Then, by Lemma 3.2 (ii), $a, b$ are in $N=C$, so that $(a, b)=0$, a contradiction.

The significance of the following lemma will become apparent later:

LEMMA 3.3. Let $R$ be an alternative ring in which $(x, y)=0$ if and only if $(x, y, R)=0$. Let $R$ contain elements $a, b$ such that $(a, b)$ is neither zero nor a divisor of zero. Then a necessary and sufficient condition that the element $k$ of $R$ belong to the centre $C$ of $R$ is that $(k, a)$ $=(k, b)=0$.

Proof. Let $K$ be the set of all $k$ in $R$ such that $(k, a)=(k, b)=0$. Let $S$ be the set of all $s$ in $R$ such that $(K, s)=0$. Clearly $C \subset K$ and $a, b \in S$. Also, since $(x, y)=0$ implies $(x, y, R)=0$,

$$
(K, S, R)=0 \text {. }
$$

By (3.1), for all $x, y$ in $R, 0=(x y, k, s)=y(x, k, s)+(y, k, s) x$ $+f(x, y, k, s)=f(x, y, k, s)$, or

$$
f(K, S, R, R)=0 \text {. }
$$

By (3.2), (2.8), (3.1), $0=3 f\left(k, s, s^{\prime}, x\right)=\left(k,\left(s, s^{\prime}, x\right)\right)-\left(s,\left(s^{\prime}, x, k\right)\right.$ $+\left(s^{\prime},(x, k, s)\right)-\left(x,\left(k, s, s^{\prime}\right)\right)=\left(k,\left(s, s^{\prime}, x\right)\right)$. Hence $(K,(S, S, R))=0$,

$$
(S, S, R) \subset S \text {. }
$$

Again, by (2.6), (3.1), $\left(s s^{\prime}, k\right)=s\left(s^{\prime}, k\right)+(s, k) s^{\prime}+3\left(s, s^{\prime}, k\right)=0$, or

$$
S S \subset S \text {. }
$$

Next, $(s x, y, k)=(x, y, k) s+x(s, y, k)+f(s, x, y, k)$. Hence, by (3.1), (3.2), and similarly,

$$
\begin{aligned}
& (s x, y, k)=(x, y, k) s, \\
& (x s, y, k)=s(x, y, k) .
\end{aligned}
$$

Using (3.3), (3.1), (3.5), (3.6), we get $0=\left(\left(s, x, s^{\prime}\right), y, k\right)=\left(s x \cdot s^{\prime}, y, k\right)$ $-\left(s \cdot x s^{\prime}, y, k\right)=s^{\prime}[(x, y, k) s]-\left[s^{\prime}(x, y, k)\right] s=-\left(s^{\prime},(x, y, k), s\right)$, or

$$
((K, R, R), S, S)=0 \text {. }
$$


In the same manner, using also (3.4), (3.7), we get $0=\left(\left(s, s^{\prime}, x\right), y, k\right)$ $\left.=\left(s s^{\prime} \cdot x, y, k\right)-\left(s \cdot s^{\prime} x, \quad y, k\right)=\left(\begin{array}{lll}x, & y, k\end{array}\right) \cdot s s^{\prime}-\left[\begin{array}{lll}x, & y, & k\end{array}\right) s^{\prime}\right] s$ $=(x, y, k)\left(s, s^{\prime}\right)$, or $(K, R, R)(S, S)=0$. However, the nonzero element $(a, b)$ of $(S, S)$ is not a divisor of zero. Therefore $(K, R, R)=0$ Since $(x, y, R)=0$ implies $(x, y)=0$, we also have $(K, R)=0$. Hence $C \subset K \subset C, K=C$. This completes the proof of Lemma 3.3.

4. Proof of the main theorem. We begin with a lemma.

LEMMA 4.1. Let $R$ be an alternative ring without divisors of zero and of characteristic not 2, generated by three elements $x, y, z$ such that $(x, y, z)$ $\neq 0$. Let $C$ be the centre of $R$. Then: (i) $p x^{2}-q x+r=0$ for $p, q, r$ in $C$, $p \neq 0$; (ii) $(x, y) \neq 0$.

Proof. (i) Write $u=(x, y, z), v=\left(x^{2}, y, z\right)=x u+u x, w=(x, x y, z)$ $=u x$. Then $u^{2} x^{2}-(u v) x+w^{2}=u^{2} x^{2}-\left(u x u+u^{2} x\right) x+(u x)^{2}$, or

$$
u^{2} x^{2}-(u v) x+w^{2}=0 .
$$

We prove (i) by showing that $u^{2}, u v, w^{2}$ are in $C$. If $S=x \cup_{y} \cup_{z}$, then $S$ generates $R$, and we may apply Lemma 3.1. By Lemmas 2.4, 2.5, $\left(u^{2}, S\right)=\left(u^{2}, S, S\right)=0$; hence $u^{2} \in C$. Since, by Lemma 2.2, $v=\left(x^{2}, y, z\right)=(x, x y+y x, z)=(x, y, x z+z x)$, a like argument shows that $v^{2}$ lies in the centres of the subrings generated by $x^{2}, y, z$, by $x, x y+y x, z$ and by $x, y, x z+z x$ respectively. In particular, $\left(v^{2}, S\right)$ $=\left(v^{2}, S, S\right)=0$, so $v^{2} \in C$. Similarly, since $u+v=\left(x+x^{2}, y, z\right)$ $=(x, y+x y+y x, z)=(x, y, z+x z+z x),(u+v)^{2} \in C$. Since $u^{2} \in C, u v$ $=u(x u+u x)=u x u+u^{2} x=v u$; hence $(u+v)^{2}=u^{2}+v^{2}+2 u v$. Thus $2 u v$ $\in C, 2(u v, R)=0=2(u v, R, R)$. Since $R$ has characteristic not 2 , $u v \in C$. Again, since $w=(x, x y, z)=(x, y, x z),\left(w^{2}, S\right)=\left(w^{2}, x, y\right)$ $=\left(w^{2}, x, z\right)=0$. Also, by $(4.1)$, since $u^{2}$ and $u v=v u$ are in $C,\left(w^{2}, y, z\right)$ $=(u v \cdot x, y, z)-\left(u^{2} x^{2}, y, z\right)=u v \cdot(x, y, z)-u^{2}\left(x^{2}, y, z\right)=u v u-u^{2} v=0$. Therefore $\left(w^{2}, S, S\right)=0, w^{2} \in C$, and the proof of (i) is complete. By a slightly more difficult proof, it may be shown that (i) also holds for characteristic 2.

(ii) Since the triples $y, z, x$ and $x+y, y, z$ also generate $R$, it is now clear that we have $p x^{2}-q x+r=0, p y^{2}-q^{\prime} y+r^{\prime}=0, p(x+y)^{2}$ $-q^{\prime \prime}(x+y)+r^{\prime \prime}=0$ for $p, q, r, q^{\prime}, r^{\prime}, q^{\prime \prime}, r^{\prime \prime}$ in $C, p \neq 0$. Subtracting the first two equations from the third, we get $p(x y+y x)-s x-t y$ $+c=0$ for $s, t, c$ in $C$. Assume that $(x, y)=0$. Then $y x=x y, s x-c$ $=2 p x y-t y$, and $0=(s x-c, z, x)=(2 p x y-t y, z, x)=2 p(y, z, x) x$ $-t(y, z, x)=u(2 p x-t)$. Since $u \neq 0,2 p x=t$. But then $0=(t, y, z)$ $=(2 p x, y, z)=2 p u$, a contradiction. Therefore $(x, y) \neq 0$. Not only the proof but also (ii) fails for characteristic 2; every Cayley-Dick- 
son division algebra of characteristic 2 can be generated by three pairwise commutative elements.

THEOREM 4.1. Let $R$ be an alternative ring without divisors of zero and of characteristic not 2, which is not associative. Then, if $C$ is the centre of $R$, every element $x$ of $R$ satisfies a quadratic equation $p x^{2}-q x$ $+r=0$ with $p, q, r$ in $C, p \neq 0$.

Proof. By the corollary to Theorem 3.1, $(x, y, R)=0$ implies $(x, y)=0$. By Lemma $4.1,(x, y, R) \neq 0$ implies $(x, y) \neq 0$. Hence the hypotheses of Lemma 3.3 are verified. If $x$ is not in $C,(x, R, R) \neq 0$, by Theorem 3.1. Therefore $(x, y, z) \neq 0$ for some $y, z$, and, by Lemma 4.1, $p x^{2}-q x+r=0$ for $p, q, r$ in the set $K$ of all $k$ such that $(k, x)$ $=(k, y)=0, p \neq 0$. Since also $(x, y) \neq 0$, we see from Lemma 3.3 that $p, q, r$ are in $C$. If $x$ is in $C$, take $q=2 p x, r=p x^{2}$ for any nonzero $p$ of $C$.

In particular, $C$ contains a nonzero element, and we may imbed $R$ in the central quotient ring $R / / C$ (see $\$ 1$ ). This yields the following corollary:

CoRollary. Under the hypotheses of Theorem 4.1, $R / / C$ is a notassociative alternative ring with unit, without divisors of zero, of characteristic not 2 , and with the field $F=C / / C$ as centre. Moreover, every element $x$ of $R / / C$ satisfies a quadratic equation $x^{2}-\alpha x+\beta=0$ with $\alpha, \beta$ in $F$.

In a recent paper [2] A. A. Albert proved a theorem which may be stated as follows:

THEOREM 4.2. Let $R$ be an alternative ring with unit, containing in its centre a field $F$ of characteristic not 2 such that, if $x$ is in $R$ but not in $F, x$ generates a quadratic field $F(x)$ over $F$. Then $R$ is an algebra of order 1, 2, 4 or 8 over $F$, order 8 corresponding to the case that $R$ is not associative.

The hypotheses of Theorem 4.2 apply to the ring $R / / C$ of the above corollary. Hence $R / / C$ is an algebra of order 8 over $F$. Moreover $R / / C$ has no divisors of zero and hence is a division algebra. This proves Theorem A (see $\$ 1$ ). Corollaries 1, 2 are immediate. As for Corollary 3, let the alternative ring $R$ be ordered. Since $a>0$, $b>0$ imply $a+b>0, a b>0, R$ has characteristic 0 and no divisors of zero. Assume that $R$ is not associative; then, by Theorem $\mathrm{A}$, $R / / C$ is a Cayley-Dickson algebra. On the one hand, $R / / C$ can be ordered by defining $x / c>0$ provided $c x>0$. On the other hand, $R / / C$ contains an (unorderable) quaternion algebra and hence cannot be ordered. In view of this contradiction, $R$ must be associative.

5. Geometrical applications. Let $\pi$ be a projective plane subject 
(tentatively) only to the axioms of incidence. If $A, B, C$ are three distinct, collinear points, the following is one of several definitions of a "harmonic conjugate" $D$ of $C$ with respect to $A, B:$ Let $A^{\prime}, B^{\prime}, C^{\prime}, D^{\prime}$ be a quadrangle (of points no three of which are collinear and none of which lies on the line $A B$ ) such that each of the triples $A B^{\prime} C^{\prime}, B C^{\prime} A^{\prime}$, $C A^{\prime} B^{\prime}, A A^{\prime} D^{\prime}, B B^{\prime} D^{\prime}$ consists of collinear points, and let $C^{\prime} D^{\prime}$ meet $A B$ in $D$. By the Theorem of the Complete Quadrilateral we mean the following pair of geometric axioms: (i) $D$ is independent of the choice of $A^{\prime}, B^{\prime}, C^{\prime}, D^{\prime}$; (ii) $D \neq C$. Part (i) is a consequence of Desargues' Theorem and (ii) asserts that no complete quadrangle has collinear diagonal points. We may now state:

TheOREM B. If a projective plane $\pi$ satisfies the Theorem of the Complete Quadrilateral, then (and only then) some coordinate ring $R$ of $\pi$ is an alternative division ring of characteristic not 2. In addition, every coordinate ring of $\pi$ is isomorphic to $R$; otherwise put, the collineation group of $\pi$ is transitive on quadrilaterals. And finally, either $\pi$ is Desarguesian or $R$ is a Cayley-Dickson algebra over its centre.

THEOREM C. Every linearly ordered projective plane which satisfies the Theorem of the Complete Quadrilateral is Desarguesian.

In a series of seven papers $[5,6,7,8,9,10,11]$ Ruth Moufang considers the influence of various axioms on projective planes. In [5] she mentions (i), (ii). In $[6,8,9]$ she uses either (i) or an equivalent (special Desargues' Theorem) $D_{9}$, together with Hilbert's (affine) axioms of order; the latter imply (ii). In [7] she asserts (i) or $D_{9}$ but tacitly uses (ii) to ensure that $A$ is the harmonic conjugate of $B$ with respect to $C, D$. In $[10,11]$, (i) and (ii) enter only implicitly. When order is used, her work leads to an ordered alternative division ring; by Theorem A, Corollary 3, the ring is associative, giving Theorem C. With order omitted she obtains the first sentence of Theorem $\mathrm{B}$ (ignoring characteristic 2 , which must be excluded because of (ii)).

In [4], Marshall Hall gives another geometric characterization of alternative division rings in terms of his Theorem $\mathrm{L}$ (a special Desargues' Theorem). Characteristic 2 plays no special rôle. Hall also gives a necessary and sufficient condition (unproved but easily demonstrated) that all alternative division rings defining the same plane should be isomorphic. For Cayley-Dickson algebras this condition had previously been verified by R. D. Schafer [12]. Hence, in view of Theorem A, Corollary 2, we have the last two sentences of Theorem B. This completes the discussion of Theorems B, C. 


\section{APPENDIX I. Associativity theOREMS}

A subset $A$ of an alternative ring $R$ is called associative if $(A, A, A)$ $=0$; for example, let $A$ consist of two elements $a, b$. And $A$ is called maximal associative provided $(x, A, A)=0$ if and only if $x$ is in $A$. Using the well-ordering axiom or Zorn's Lemma, one can easily verify that every associative subset is contained in at least one maximal associative subset.

Theorem I.1. Let $R$ be an alternative ring in which $3 f(w, x, y, z)$ $=0$ implies $f(w, x, y, z)=0$. Then every maximal associative subset $A$ of $R$ is a subring of $R$.

Proof. If $w, x, y, z$ are in $A,(2.8)$ and the hypothesis on $R$ yield $f(w, x, y, z)=0$. Then (2.7) gives $(w x, y, z)=0$. Hence $A A \subset A$. By the linearity of the associator, $A$ is closed under subtraction, and the proof is complete.

An (unpublished) example of an alternative ring $R$ for which the conclusion of Theorem I.1 fails has been constructed by M. F. Smiley [14]. Therefore the following theorems have some point. But it should be observed that Theorem I.1 is the definitive associativity theorem for the wide class of rings satisfying its hypothesis.

Theorem I.2. Let $A, B, C$ be subsets of an alternative ring $R$ such that $(A, A, R)=(B, B, R)=(C, C, R)=(A, B, C)=0$. Then the subset $D=A \cup B \cup C$ is contained in an associative subring of $R$.

Corollary. Any two elements $a, b$ of $R$ (or any three elements $a, b, c$ such that $(a, b, c)=0$ ) are contained in an associative subring of $R$.

RemarK. Max Zorn [15] proves the case $C=B$ (generalized Theorem of Artin). Moufang [11] proves the case $A=a, B=b, C=c$ simultaneously for alternative division rings and Moufang loops. Smiley [13] modifies Moufang's proof to apply to general alternative rings. All of these proofs use mathematical inductions, which are particularly complex in the case of the latter two authors.

Proof. Let $a, a^{\prime}, a^{\prime \prime}$ denote elements of $A$, and similarly for the other subsets occurring in the proof. Since $0=\left(r r^{\prime}, a, a^{\prime}\right)=r^{\prime}\left(r, a, a^{\prime}\right)$ $+\left(r^{\prime}, a, a^{\prime}\right) r+f\left(r, r^{\prime}, a, a^{\prime}\right)=f\left(a, a^{\prime}, r, r^{\prime}\right)$ (and similarly),

$$
f(A, A, R, R)=f(B, B, R, R)=f(C, C, R, R)=0 .
$$

Let $K$ be the set of all $k$ in $R$ such that $(D, D, k)=f(A, B, C, k)=0$. In view of (1) and the hypotheses, $D \subset K$. In addition, 


$$
(D, D, K)=0=f(D, D, D, K) .
$$

By (2), $\left(d d^{\prime}, d^{\prime \prime}, k\right)=d^{\prime}\left(d, d^{\prime \prime}, k\right)+\left(d^{\prime}, d^{\prime \prime}, k\right) d+f\left(d, d^{\prime}, d^{\prime \prime}, k\right)=0$, or $(D D, D, K)=0$. This, (2), and (1), for $x$ in $D \cup D D$, give $\left(a k, a^{\prime}, x\right)$ $=k\left(a, a^{\prime}, x\right)+\left(k, a^{\prime}, x\right) a+f\left(a, k, a^{\prime}, x\right)=0$ or $(D, A, A K)$ $=(D D, A, A K)=0$. Therefore $0=\left(b c, a, a^{\prime} k\right)=c\left(b, a, a^{\prime} k\right)+\left(c, a, a^{\prime} k\right) b$ $+f\left(b, c, a, a^{\prime} k\right)=f\left(a, b, c, a^{\prime} k\right)$. Thus, and by symmetry, $f(A, B, C, D K)$ $=0$. Again, by (2), since $D \subset K,\left(d k, d^{\prime}, d^{\prime \prime}\right)=k\left(d, d^{\prime}, d^{\prime \prime}\right)+\left(k, d^{\prime}, d^{\prime \prime}\right) d$ $+f\left(d, k, d^{\prime}, d^{\prime \prime}\right)=0$, or $(D, D, D K)=0$. Therefore $D K \subset K$.

If $M$ is the set of all $m$ in $K$ such that $(D, m, K)=0$ and $m K \subset K$, we have proved that $D \subset M$.

Finally, let $S$ be the set of all $s$ in $M$ such that $(s, M, K)=0$. Clearly $D \subset S$. Moreover $S$ is associative and (along with $K, M$ ) closed under subtraction. Since $S \subset M \subset K$ and $M K \subset K, 0=\left(m k, s, s^{\prime}\right)$ $=k\left(m, s, s^{\prime}\right)+\left(k, s, s^{\prime}\right) m+f\left(m, k, s, s^{\prime}\right)=f\left(m, k, s, s^{\prime}\right)$, so that $\left(s s^{\prime}, m, k\right)$ $=s^{\prime}(s, m, k)+\left(s^{\prime}, m, k\right) s+f\left(s, s^{\prime}, m, k\right)=0$. Therefore $(S S, M, K)=0$. Since $S \subset M \subset K$ and $M K \subset K, S S \subset K$. Also $(S, S, K)=0$, so that $S S \cdot K=S \cdot S K \subset K$. And $(S S, M, K)=0$ implies $(S S, D, K)=0$, so that $S S \subset M$. Therefore $S S \subset S$, and the proof is complete. As for the corollary, set $A=a, B=C=b$ or $A=a, B=b, C=c$ according to the case.

It is clear on the basis of Zorn's Lemma that every associative subring of an alternative ring is contained in at least one maximal associative subring.

THEOREM I.3. Let $A$ be an associative subring of the alternative ring $R$, and let $B$ be a subset of $R$ such that $(A, A, B)=(B, B, R)=0$. Then the subset $D=A \cup B$ is contained in an associative subring of $R$.

COROLlaRy. Every maximal associative subring of $R$ is a maximal associative subset.

Proof. The proof is so similar to that of Theorem I.2 that we may omit some details. Let $K$ be the set of all $k$ in $R$ such that $(D, D, k)=0$. Since $(B, B, R)=0, f(B, B, R, R)=0$. Since $A A \subset A$ $C D, 0=(A A, D, K)=f(A, A, D, K)$. Therefore $f(D, D, D, K)=0$. Moreover $D \subset K$ and hence $(D K, D, D)=0$. Thus $D \subset M$, where $M$ is the set of all $m$ in $K$ such that $(D, m, K)=0$ and $m K \subset K$. If $S$ is the set of all $s$ in $M$ such that $(s, M, K)=0$, we see as before that $S$ is an associative subring containing $D$. As for the corollary, let $A$ be a maximal associative subring of $R$ and let $B=b$ where $(A, A, b)=0$. By Theorem I.3, $A \subset A \cup b \subset S$ where $S$ is an associative subring. Hence $A=A \cup b, b$ is in $A$. 


\section{BibliogRAPHY}

(For further references see in particular [3] and [14].)

1. A. A. Albert, On ordered algebras, Bull. Amer. Math. Soc. vol. 46 (1940) pp. $521-522$.

2. - Absolute-valued algebraic algebras, Bull. Amer. Math. Soc. vol. 55 (1949) pp. 763-768.

3. L. E. Dickson, On quaternions and their generalizations and the history of the eight square theorem, Ann. of Math. (2) vol. 20 (1919) pp. 155-171.

4. Marshall Hall, Projective planes, Trans. Amer. Math. Soc. vol. 54 (1943) pp. 229-277.

5. Ruth Moufang, Zur Struktur der projektiven Geometrie der Ebene, Math. Ann. vol. 105 (1931) pp. 536-601.

6. - Die Einfihhrung der idealen Elemente in die ebene Geometrie mit Hilfe des Satzes vom vollständigen Vierseit, Math. Ann. vol. 105 (1931) pp. 579-778.

7. - Die Schnittpunktsätze des projektiven speziellen Filnfecksnetzes in ihrer Abhängigkeit voneinander (Das A-Netz), Math. Ann. vol. 106 (1932) pp. 755-795.

8. - Ein Satz über die Schnittpunktsätze des allgemeinen Fünfecksnetzes (Das $(A, B)$-Netz), Math. Ann. vol. 107 (1933) pp. 124-139.

9. - Die Desarguesschen Sätze vom Rang 10, Math. Ann. vol. 108 (1933) pp. $296-310$.

10. - Alternativkörper und der Satz vom vollständigen Vierseit $\left(D_{9}\right)$, Abh. Math. Sem. Hamburgischen Univ. vol. 9 (1933) pp. 207-222.

11. —, Zur Struktur von Alternativkörpern, Math. Ann. vol. 110 (1934) pp. 416-430.

12. R. D. Schafer, Alternative algebras over an arbitrary field, Bull. Amer. Math. Soc. vol. 49 (1943) pp. 549-555.

13. M. F. Smiley, The radical of an alternative ring, Ann. of Math. (2) vol. 49 (1948) pp. 702-709.

14. - Some questions concerning alternative rings, Bull. Amer. Math. Soc. vol. 57 (1951) pp. 36-43.

15. Max Zorn, Theorie der alternativen Ringe, Abh. Math. Sem. Hamburgischen Univ. vol. 8 (1930) pp. 123-147.

UNIVERSITY OF WISCONSIN AND

University of Chicago 